\title{
Una Company Town francesa en el desierto de la Baja California: Compagnie Du Boleo, Santa Rosalía 1885
}

SELECCIÓN VII SEMINARIO INTERNACIONAL DE INVESTIGACIÓN EN URBANISMO

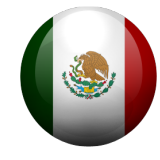

\section{Enrique Esteban Gómez Cavazos}

Arquitecto por la Universidad Autónoma de Nuevo León, en Monterrey, México. Estudiante de doctorado en Urbanismo por el Programa de Doctorado Universitat Politécnica de Catalunya, en Barcelona, España. Ensenada [Baja California] México. <eecavazos@hotmail.com>.

\section{Resumen}

Este artículo se centra en el alcance urbano y arquitectónico de la inversión extranjera en la península de Baja California en México y refuerza la idea de la modernización de un territorio inhóspito rico en recursos minerales. Se analiza la construcción de una ciudad que desarrolla un nuevo tipo de urbanismo e industria a través de la concentración de materia prima, fuerza de trabajo y la exportación de productos terminados. La consolidación de la compañía minera da lugar a la fundación de una nueva ciudad: Santa Rosalía, cuyo desarrollo urbano se basa en la conexión directa entre residencia y trabajo. La ciudad presenta una segregación social evidente junto a condiciones laborales difíciles, deudoras de lo que trae la Revolución Industrial en el país. A mediados del siglo XX la ciudad se encuentra en su apogeo económico pero comienzan ya los agotamientos minerales en el sistema de poblaciones y en las minas más productivas. La entrada al periodo post-industrial, la quiebra de la compañía y el abandono suponen una regeneración urbana a partir del declive económico para reconocer y recuperar hoy el valioso patrimonio industrial que aún existe y que actualmente está en deterioro.

\section{Palabras clave}

Company town, península de Baja California, industrialización, patrimonio.

\section{A french company town in Baja California desert: Compagnie Du Boleo, Santa Rosalía 1885}

\begin{abstract}
This article focuses on the urban and architectural scope of foreign investment in the peninsula of Baja California in Mexico and strengthens the idea of modernization in an inhospitable territory rich in mineral resources. Here we analyze the construction of a city that develops into a new kind of urbanism and industry by concentrating raw material, labor, and finished products for export. The consolidation of the mining company leads to the foundation of a new city: Santa Rosalía, whose urban development follows a common pattern based on the direct connection between residence and work. Santa Rosalía shows a clear social segregation with difficult working conditions, which were characteristic of the industrial revolution in the country. In the mid-twentieth century, the city is at its economic peak, but mineral depletions start appearing in the most productive mines and the population systems. The entrance to the post-industrial period, the company's bankruptcy, and its abandonment call for an urban regeneration from economic decline in order to acknowledge and recover valuable industrial heritage that still exists yet is currently in decay.
\end{abstract}

\section{Keywords}

Company town, Baja California peninsula, industrialization, heritage. 


\section{Introducción}

El Norte de México puede ser considerado como un territorio que se ha dedicado, sobre todo, a complementar la gigantesca economía de los Estados Unidos. No es un fenómeno nuevo, puede detectarse ya desde 1850 cuando el Río Bravo y el desierto se convierten en nueva línea divisoria. La industrialización es escenario de desarrollos regionales que nacen con insistente frecuencia y vive su mayor modernización a manos de capitalistas norteamericanos y europeos, gracias a las nuevas y pujantes ciudades industriales en un territorio árido que se convierte en potencia mundial durante el siglo XX (Cerutti, 2008).

La península de Baja California en México comprende dos litorales, uno en el Pacífico y otro dentro del Golfo de California. Es un territorio donde los yacimientos cupríferos que mantuvieron la actividad de El Boleo fueron descubiertos a mitad de la península en 1868 por un ranchero del vecino pueblo de Santa Águeda. En 1870 Eduard Cumminge, geólogo francés rindió el primer informe a Europa sobre las posibilidades del mineral y en 1885 se funda con capital Rothchild en París la Compagnie Du Boleo que se encargó del trazado, el diseño y la construcción de la ciudad.

La presente investigación pretende indagar en la historia urbanística a partir del estudio de factores como los procesos de industrialización en México, la innovación y la construcción de establecimientos fabriles, tomando de referencia el surgimiento de ciudades de nueva fundación. La traza urbana y la ubicación calculada del sistema de minas nos ayudan a definir la ocupación del área. El puerto industrial es el lugar donde se encuentra la mayoría de las evidencias arquitectónicas y es ahí donde se enfoca el análisis.

El trabajo reconoce la importancia de proteger las edificaciones que modernizaron esta parte del territorio mexicano y propone posibles oportunidades para su valorización. Este es el objetivo principal y punto de partida para proyectar a futuro un nuevo desarrollo de la región basada en su propia historia y memoria de trabajo.

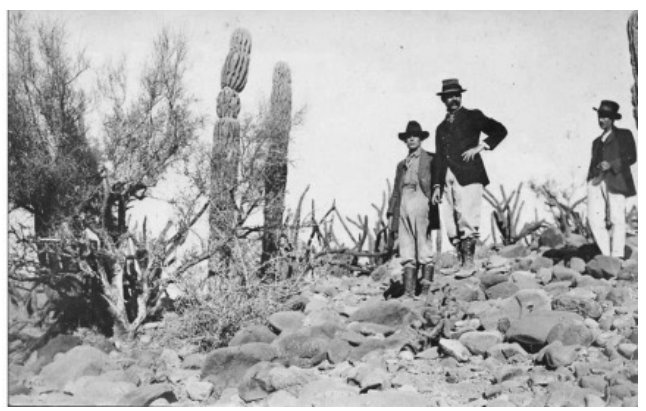

Figura 1. León Diguet químico francés publica en 1912 una serie de planos cartográficos de algunas partes de la península de California. Fuente: Territorio de Baja California.

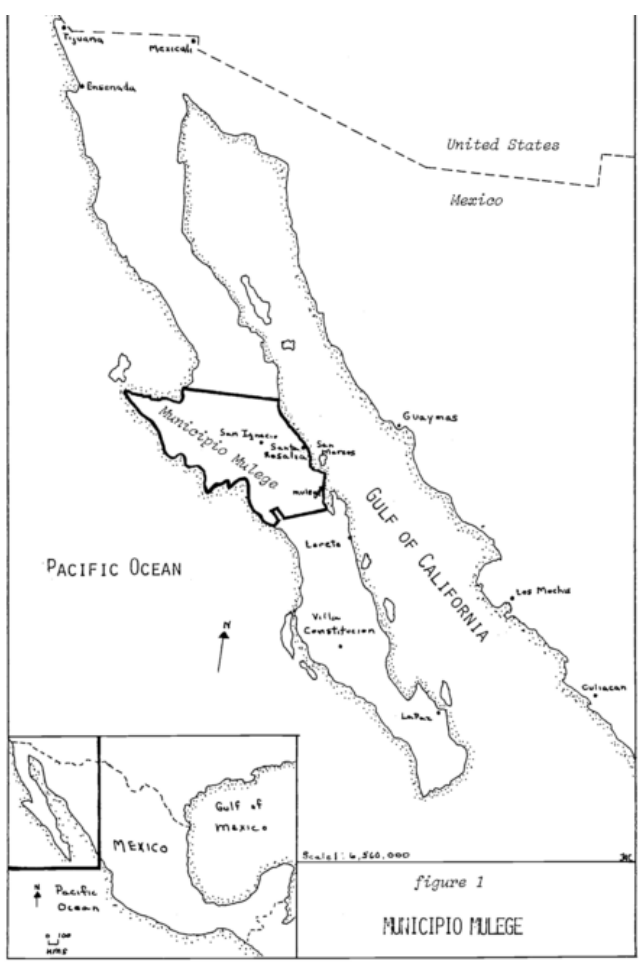

Figura 2. Ubicación del caso de estudio. Fuente: Mapa de Judith Cichowicz. The economic evolution of a mining community: Santa Rosalia, Baja California Sur, Mexico. The Departament of Geography Oregon State University.

\section{Contexto territorial e inicios de la ciudad industrial}

De 1876 a 1911 con el Porfiriato (periodo de gobierno del presidente Porfirio Díaz) el poder Ejecutivo tiene mayor espacio político, aparecen grandes e importantes cambios legales en relación con la actividad minera. En primera instancia por lo que se refiere al marco legal de la minería, el Porfiriato marcará una tajante diferencia con sus antecesores. Pronto se sacudirá una legislación obsoleta que databa desde 1783, año en que se publicaron las ordenanzas de minería que chocaban con el proyecto liberal del Estado mexicano encaminando a establecer las bases jurídicas y la infraestructura productiva que permitirían al país crecer hacia afuera en un periodo de estabilidad económica. (Romero, 1991) 


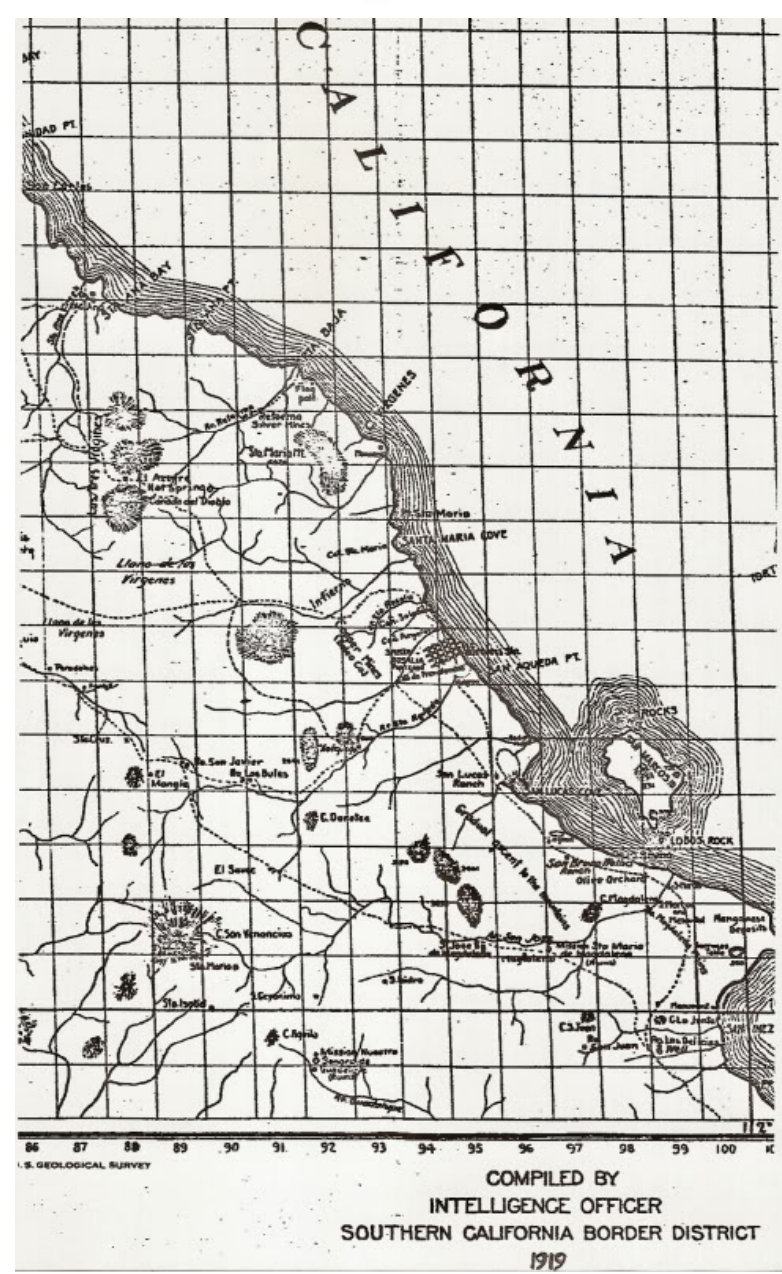

Figura 3. Localización del litoral peninsular. Fuente: Mapa de 1919 realizado por la oficina de inteligencia del Sur de California y su distrito fronterizo de Estados Unidos.

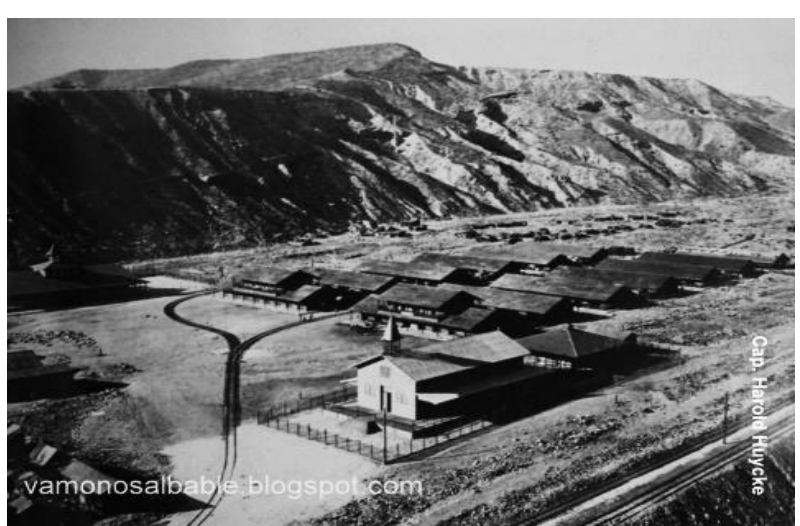

Figura 4. El poblado a finales del siglo XIX. Fuente: Fotografía del Capitán Harold D. Huycke.

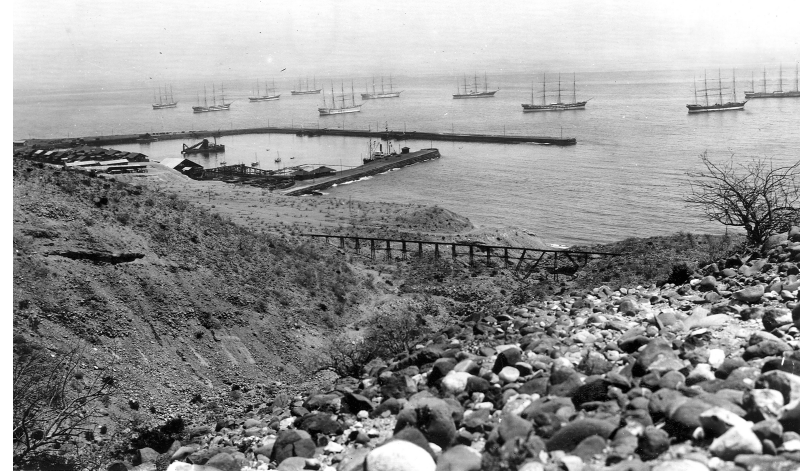

Figura 5. Fotografía del puerto de Santa Rosalía a inicios del siglo XX. Fuente: San Diego History Center. Colección de The Journal of San Diego History, invierno de 1989, Volumen 35, Numero 1.

Una política ferroviaria que comprometió al Estado a ayudar a la construcción de las vías férreas y que además concedió un derecho de vía de 60 metros a cada lado de las líneas. Área que, en caso de contener yacimientos minerales, era concedida a las empresas constructoras, entregándoles también los terrenos necesarios para levantar edificios administrativos, viviendas para trabajadores, talleres, fabricas, patios, etcétera.

La creación de ciudades de nueva fundación y la explotación de mineral como razón prioritaria, constituye un capítulo específico de la urbanística del siglo XIX. En su modalidad más genérica recibe el nombre de ciudad industrial: Industrial Village en Inglaterra, Cite Ouvriere en Francia, Arbeiter Siedlungen en Alemania, Colonia Industrial en España y company town en Estados Unidos (Garcés, 1988).

El asentamiento nace en la mitad de la península de California donde la compañía francesa diseña una ciudad minera entre dos montañas frente al Golfo de California. En 1886 se instala el primer horno "water jacket" para abastecer a la fundición, se construye un pequeño puerto de madera que dura poco, a consecuencia de las tempestades, en los siguientes años se formaliza la construcción de un puerto industrial de noventa y cinco metros de boca construido de acero y escoria que comunica la nueva ciudad con el resto de los puertos del pacífico mexicano y a las vías de ferrocarril en la costa sonorense. En las próximas décadas se consolida la primera ciudad industrial para la extracción y procesamiento del cobre en México.

Cabe señalar que los navíos aún de velas a finales del siglo XIX, son el transporte para la madera que se usa para la construcción de la ciudad, traída de San Francisco, California, lugar de grandes bosques. 


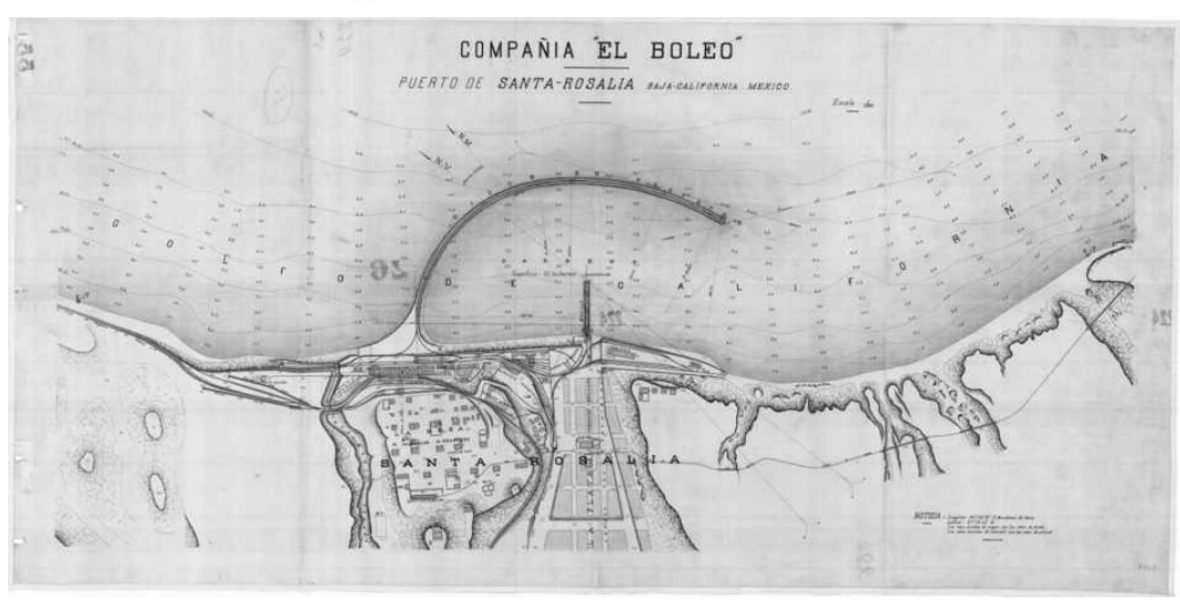

Figura 6. Plano del puerto de Santa Rosalía Baja California Sur, MAMOYB, Compañía El Boleo. Fuente: Mapoteca Nacional Manuel Orozco y Berra, colección: General, autor: desconocido, año: no determinado, escala: 1:20,000.

En esos años San Francisco crecía aceleradamente a consecuencia de la fiebre del oro, consolidándose en poco tiempo en un puerto importante. Fue la ciudad más poblada de las Californias con cerca de los 60.000 habitantes en 1860, antes de que se completara la línea de ferrocarril interoceánico de América. Los barcos, para no regresar vacíos a Santa Rosalia aprovechaban para traer la madera de la alta california, después de haber comerciado a muy buen precio todo el cobre del desierto baja californiano.

\section{La consolidación del puerto industrial en los umbrales del siglo XX}

En la frontera entre México y Estados Unidos al Norte del país en 1883 la Southern Pacific Railroad extiende sus conexiones ferroviarias con un ramal en el estado de Sonora llegando hasta el puerto de Guaymas. En estos mismos años ya se tienen registros del rico mineral de cobre en la vecina península de Baja California. En los próximos años ese mismo ramal norteamericano enlaza las ciudades mineras de Cananea y Nacozari para uso exclusivo de acarreo de minerales. Cabe mencionar que antes de que las compañías extranjeras llegaran a territorio mexicano ya existían en estos lugares pequeños asentamientos mineros. Algunos de ellos fueron después de 1810 asentamientos abandonados, rodeados de barrios mineros con una población muy pequeña.

La Cananea Consolidated Cooper Company (4C), labora de 1899 a 1906, operando 16 minas. La importancia de la minería en la ciudad de Cananea se ve en el aumento de su población, ya que en el año 1891 tiene cien habitantes. En 1900 cuenta con 900 habitantes, y en 1906 más de 22.000, convirtiendo a esa población en la más poblada e importante del estado de Sonora (Sariego, 1992). En Santa Rosalía ocurre algo similar, el poblado diseñado por la Compagnie du Boleo se convierte en la ciudad más industriosa de la península, llegando en 1900 a más de 10.000 habitantes.

La ciudad de Santa Rosalía se mantiene alejada del proceso revolucionario entre 1910 y 1920 ya que su condición geográfica de aislamiento la protege del conflicto que cambiaría para siempre el funcionamiento de la ciudad, ya que los gobiernos post revolucionarios debilitaron la injerencia de la compañía en la población. Algunos edificios cambiaron su función como por ejemplo el edificio de la escuela para trabajadores que pasó a ser el edificio del Ayuntamiento. El Gobierno mexicano avisa que el porfiriato ha acabado ya años atrás y que el Gobierno Federal tiene que hacerse presente cambiando nombres de calles y edificios. Se crea así, la municipalidad en la primera company town de la Baja California.

El desarrollo urbano aquí acomoda la industria en los yacimientos y los conecta al puerto. Se puede ver un mismo modelo de vivienda para obreros que se sitúa cerca de las fundiciones y minas, así evitaban largos desplazamientos de la mano de obra, garantizando una comunicación directa entre casas y trabajo. En relación a la cuadrícula principal de calles y manzanas, es interesante notar que obedece más a una intención de pura funcionalidad, al haberse colocado la vivienda a los lados de un eje longitudinal, no favoreciendo a la integración cívica y social, como fuera el caso de haberse agrupado alrededor de la típica plaza (Padilla, 1989). 


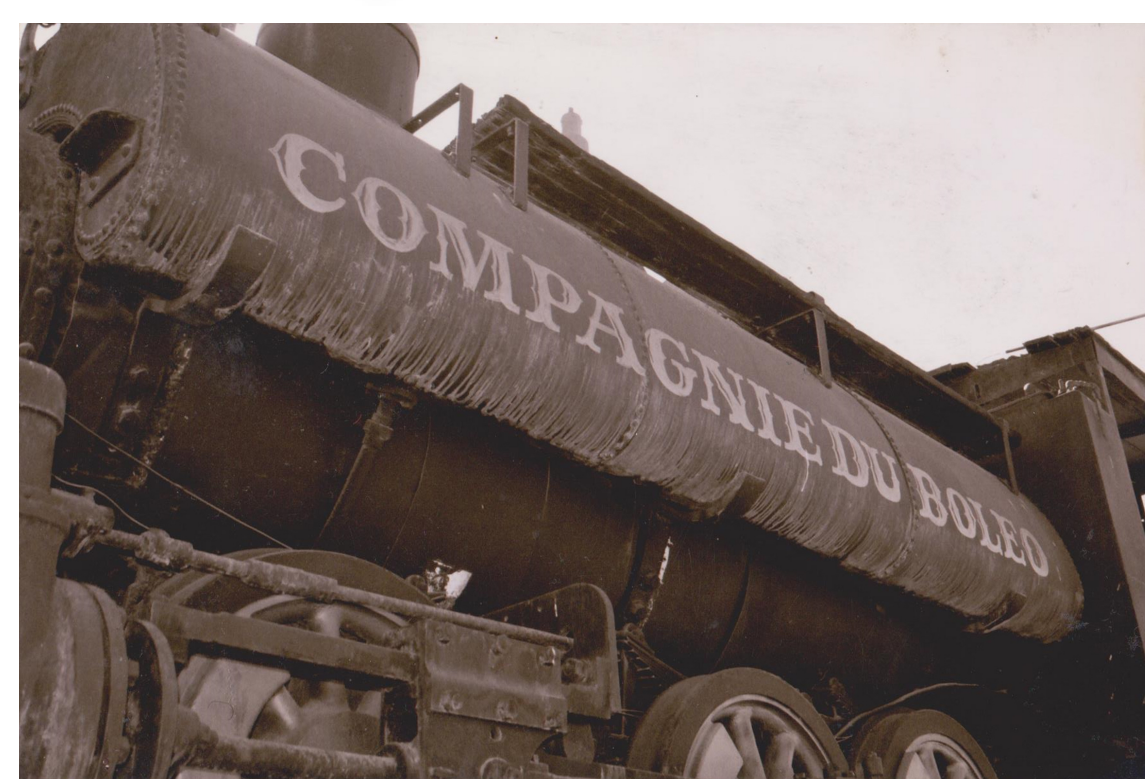

Figura 7. La construcción del ferrocarril en Santa Rosalía Baja California Sur comenzó en 1886 con las primeras vías, carros y máquinas. Estaban incluidas 3 locomotoras de vapor marca Baldwin construidas ese mismo año. Las locomotoras estaban numeradas del 1 al 3, quemaban carbón, estaban pintadas de verde olivo y llevaban los nombres individuales de Búfalo, Morueco y Toro, llevaban además, un pequeño letrero en donde se leía COMPAGNIE DU BOLEO (Romero Manuel, 1991. El Boleo: un pueblo que se negó a morir: 1885-1954. Hermosillo, Sonora. Editorial Unísono).

La característica urbana que se ve a simple vista en el área de viviendas es su regularidad en las dimensiones de calles y manzanas. Éstas están organizadas a los lados del eje longitudinal el cual se convierte en la avenida principal, no por ser la de mayor anchura con respecto a las demás ni el hecho de que se haya establecido el comercio y otra serie de actividades importantes, sinó sencillamente, por ser la calle de mayor longitud. Las actividades comerciales, sociales y religiosas se desarrollan a lo largo de una avenida paralela (Padilla, 1989).

Las otras áreas habitacionales del conjunto, no siguen los principios urbanos establecidos en el puerto de Santa Rosalía. En el área conocida como ranchería al cruzar el arroyo en la parte Suroeste, se disponen diminutas viviendas para los mineros de menor categoría. Su disposición es similar a la de la colocación de barracas en un campo militar, es decir, con carácter provisional y dejando entre ellas los espacios estrictamente indispensables para la circulación interior (Padilla, 1989).

\section{Reconociendo el legado urbanístico}

La traza urbana principal de la ciudad se asienta prácticamente sobre el lecho del río y por consiguiente, en el lugar menos indicado y más inseguro. Las otras dos áreas que componen la ciudad, "La Mesa Francia" se ubica en el cerro rocoso al Norte del acceso y la "Mesa México" al Sur, en la otra colina aledaña. Se denomina "Mesa" a los asentamientos que se encuentran en cotas altas, ambas colinas a los lados del acceso principal, aparentan ser los guardianes de la ciudad (Padilla, 1989).

El tejido urbano obedece a un diseño funcional, de manera que todas las edificaciones están orientadas alrededor de las minas y servicios, siendo el puerto la traza más potente y las demás poblaciones compuestas por las minas más productivas de la empresa. Surge así, una pequeña ciudad minera con poblaciones satélite conectadas al puerto, que cuentan también con equipamientos básicos como escuelas, comercios, viviendas, e iglesias. Las viviendas son casas de un piso, funcionales y geométricas, construidas todas ellas de madera.

El asentamiento responde a un nuevo concepto de industria importada a México, una fábrica entera, que aloja la fundición industrializada de cobre consus diferentes edificios industriales y de equipamiento, concentrando en lugares específicos la extracción del mineral, vivienda, mano de obra y el producto acabado para la exportación.

El circuito ferroviario de treinta y nueve kilómetros conectó el puerto con las poblaciones periféricas y minas más importantes de la compañía, las cuales hicieron que éstas poblaciones periféricas tuvieran más población que el mismo puerto y centro dominante donde se extiende la ciudad de madera. Fue levantada con el sistema de construcción "balloon frame" que apareció por primera vez en 1832 en la ciudad de Chicago y que se extendió principalmente a Canadá, a todo el medio oeste norteamericano y al norte de México. 


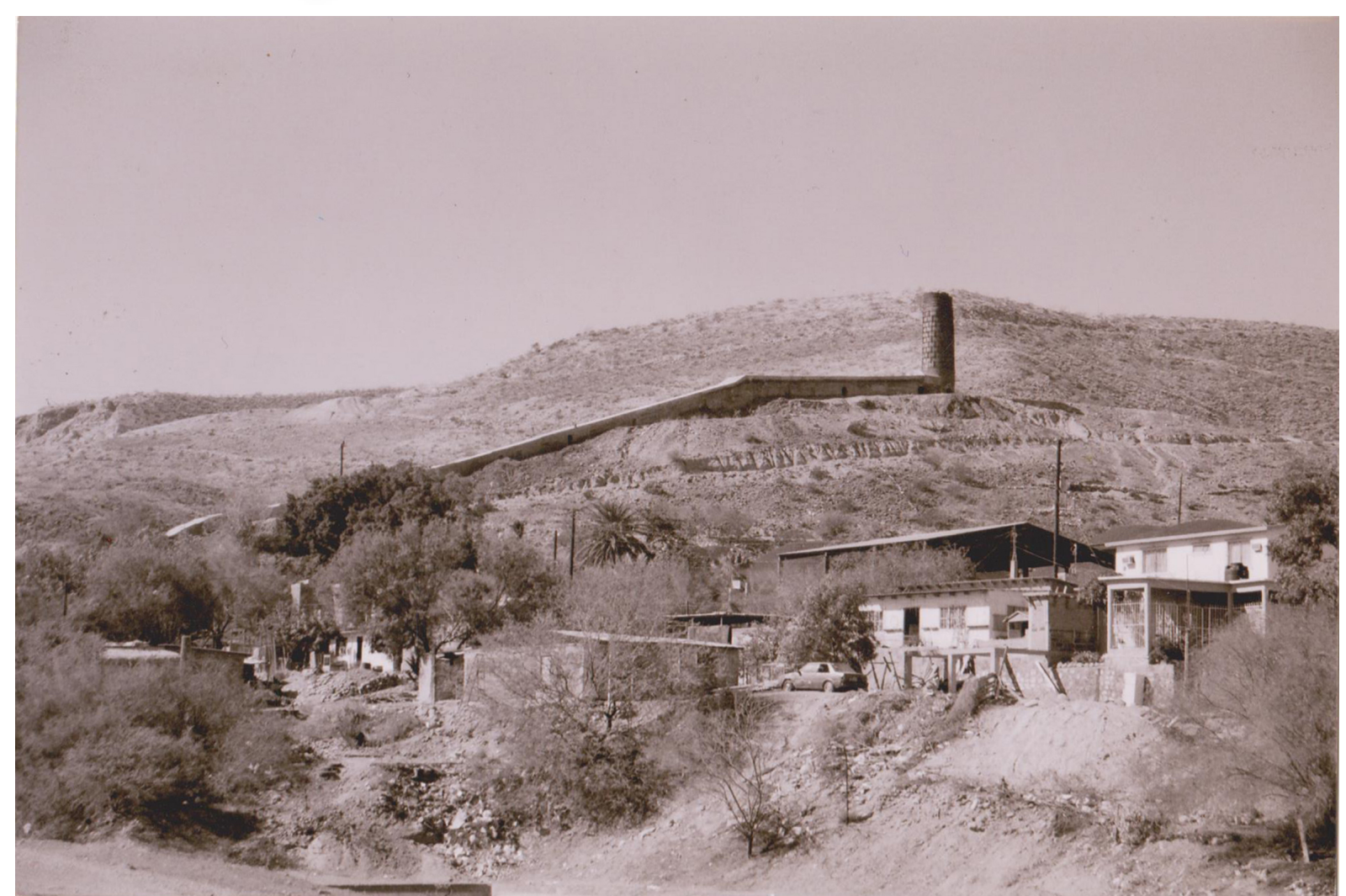

Figura 8. Las minas del Distrito de Santa Águeda en Santa Rosalía fueron muy ricas en mineral de cobre. Se basó en una alta explotación de la fuerza de trabajo de origen Yaqui, los cuales escarbaron primero en la superficie y luego en las entrañas de la tierra a través de túneles. El capital de la compañía francesa provenía de la casa Rothschild que fueron los financieros más grandes del mundo en minería industrial a finales del siglo XIX y principios del siglo XX.

La posición del norte de México, dentro de una economía de frontera que se abre con celebridad al capitalismo trae junto al ferrocarril una nueva manera de construir. Llegan nuevos materiales como la madera, el ladrillo, equipos y tecnología procedentes del medio oeste norteamericano. Al mismo tiempo, el ferrocarril se convierte en vehículo de la modernidad arquitectónica. Se pasa de la construcción con adobe a la madera, el vidrio y las laminas de zinc. Se impulsa el ya mencionado sistema constructivo balloon frame, constituido por estructuras ligeras de madera, se usan igualmente las fachadas y edificios prefabricados, consolidando rápidamente los proyectos urbanos de las nacientes poblaciones, todas las estructuras eran hechas en Chicago y vendidas por piezas en catálogos (Brown, 2009).

Las edificaciones mezclan elementos nacionales, norteamericanos y europeos. Las viviendas se componen de recibidor, sala de estar, comedor, dormitorio, cocina y baño. La pared exterior es de tabla rustica ancha, los techos de fuerte pendiente cubierta de lámina de zinc, con amplios aleros de protección con cielo falso y pisos de tabla clavados sobre vigas. Se levantan del suelo con polines de madera (Novelo, 1988).

Respecto a la Mesa México, quedan definidas perfectamente dos áreas urbanas, debido a su ubicación en la loma sur del pueblo frente al mar. El territorio es desértico y el paisaje está compuesto por varios arroyos secos que rodean la ciudad. La Delegación Municipal y las casas habitación de empleados federales importantes se ubican de manera que sean visualizadas fácilmente por las embarcaciones al aproximarse al puerto Padilla, 1989).

En la Mesa Francia cambian un poco las características urbanas. El pequeño agrupamiento de viviendas y oficinas de la compañía se dispone en ambos lados de una calle corta, pero bastante ancha, con orientación noreste - sureste. Comenzando por su ángulo mas al Sur, se construye la Dirección General del Boleo con vistas hacia el puerto y asimismo hacia el resto de la población. Por su tamaño, acentuando en sentido horizontal por la amplia vereda que rodea todo el edificio y en el plano vertical, por los techos inclinados a varias aguas y en especial por la torre del reloj colocada sobre su acceso principal, presenta una imagen urbana imponente que denota autoridad, 
disciplina, pero sobre todo vigilancia. Todo el pueblo: el puerto, la Mesa México, el acceso a la cañada y los cerros al fondo, están a la vista del ojo vigilante de la cabeza de la compañía (Padilla, 1989).

Al costado izquierdo de las oficinas se ubican las viviendas de los ingenieros y funcionarios principales, alineadas sobre la calle principal, que a pesar de ser de poca longitud, contrasta por su gran anchura. Frente a esta acera, se localizan algunos de los edificios que otorgan servicios a la comunidad, como el Hospital Civil, que atiende a todos los trabajadores de la empresa y el Hotel Francés construido a todo lujo, en su extremo opuesto (Padilla, 1989).

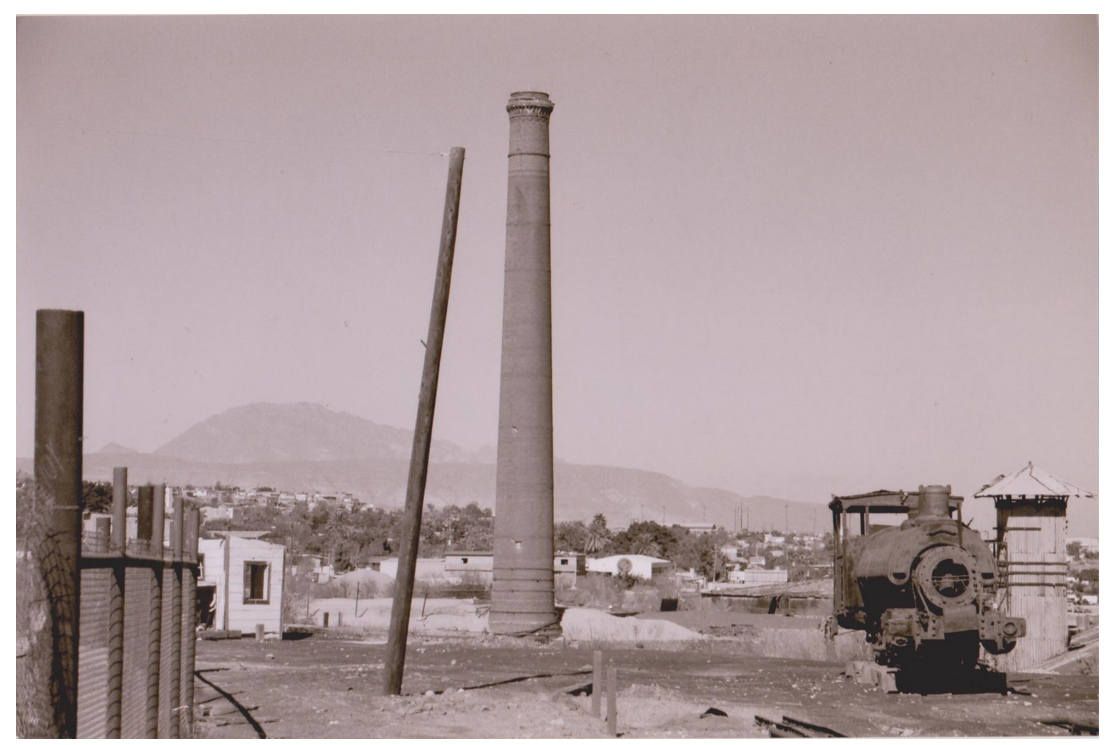

Figura 9. El ferrocarril conectaba los 3 grupos mineros de nombre apocalíptico: La Providencia, El Purgatorio y La Soledad que distaban del puerto de Santa Rosalía a 5,7 y $10 \mathrm{Km}$. La vía fue tendida en camino natural atravesando el centro del pueblo y por las orillas entre secas mesetas y omeríos hasta alcanzar los tendidos de los grupos mineros (Romero Manuel, 1991. El Boleo: un pueblo que se negó a morir: 1885-1954. Hermosillo, Sonora. Editorial Unísono). La articulación entre los poblados y el puerto hacían a la company town un mecanismo urbano de eficiencia industrial que funcionaba para extraer la mayor cantidad de cobre posible.

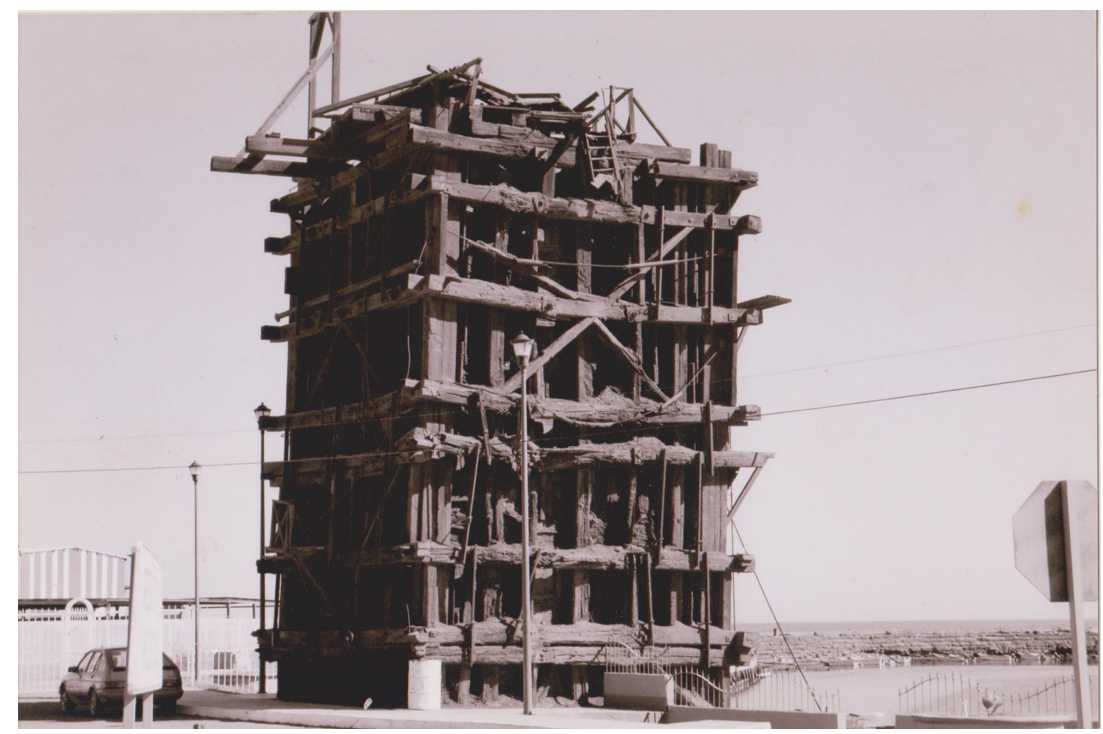

Figura 10. El Boleo sirvió para impulsar la colonización de una región de México prácticamente deshabitada. De 1876 a 1910 con el Gobierno de Porfirio Díaz se expresaba la intención de articular a la península de Baja California con el mercado nacional, los inversionistas franceses aprovecharon las óptimas condiciones políticas para dar curso a un proyecto minero que derivó en una exitosa (Romero Manuel, op. cit.,1991.) company town, que tuvo durante un largo tiempo el control económico y político de la zona en que se encontraba el mineral, realizando una explotación a gran escala de los ricos yacimientos cupríferos.

Aproximadamente en el centro de la calle se localiza la majestuosa residencia del director de la compañía. Paralelamente a esta avenida principal, se trazan dos calles donde se construyen varias casas para los empleados de confianza, pero evidentemente de menor categoría, dada la estrechez de sus dimensiones. La estrategia urbana parte aquí de la dirección de la empresa. Se establece una jerarquía: los funcionarios franceses, los mexicanos de confianza, las autoridades federales y la vivienda de $1^{\underline{a}}, 2^{\underline{a}}, 3^{\underline{a}}$ y de $4^{\underline{a}}$. Es decir, en los conjuntos se expresa un orden racional producto de la Revolución Industrial ya muy avanzada en el resto del mundo.

Sumando los centros mineros periféricos de La Soledad, El Purgatorio y La Providencia, la población es mayor que la del puerto. Esto indica la importante densidad en estos núcleos urbanos donde a los mineros se les paga un poco más que a los obreros justificando la construcción de estos pueblos 
mineros satélites, colocados a un lado de la mina y conectados por ferrocarril al puerto. Se puede concluir que Santa Rosalía es un todo articulado que funciona eficientemente hasta que se agota el mineral y estos núcleos urbanos se abandonan poco a poco quedando en la actualidad sólo el centro más consolidado frente al puerto.

La estrategia utilizada por la compañía es la de dividir la ciudad en zonas, todas ellas con iglesia, escuela, comercios, oficinas, estación de ferrocarril y viviendas. Esto muestra una preocupación por un sistema urbano que produce cobre eficientemente, lográndolo a través de un urbanismo calculado. Se separan áreas para las viviendas de obreros de la planta de fundición a las afueras de Santa Rosalía (en el arroyo que se inunda); el área para los directivos franceses del Boleo en la Mesa Francia, dominando el panorama; el área de los empleados del gobierno mexicano en la Mesa México, así toda la población se ubica cerca de su trabajo, lo que determina el planeamiento urbano, además de las jerarquías o niveles en la compañía, que también influyen en la configuración del espacio en el territorio.

La existencia de cuatro asentamientos de los que hoy apenas queda uno es un ejemplo único y completo de company town, en donde todas las partes del conjunto desempeñan un papel especial en el mecanismo total, cuyo objetivo central es extraer y procesar la mayor cantidad de cobre posible.

Para completar el urbanismo se necesita agua. Además de requerirse para la fundición, las poblaciones la traen del arroyo más grande de la región: el arroyo de Santa Águeda, por medio de pozos. Debido a la escasez de lluvia en el desierto, se utilizan bombas de vapor a través de lomas, seguramente algo increíble para su tiempo. Pero el cobre lo vale y la producción lo paga y aún así quedan buenas ganancias para los empresarios. Los trenes recorren los perímetros de los arroyos, que siempre tienen pendientes suaves. Así los ha modificado el agua durante milenios cuando llueve. Los trenes antiguos siempre buscan evitar las lomas y las recortan para evitar los cambios bruscos de

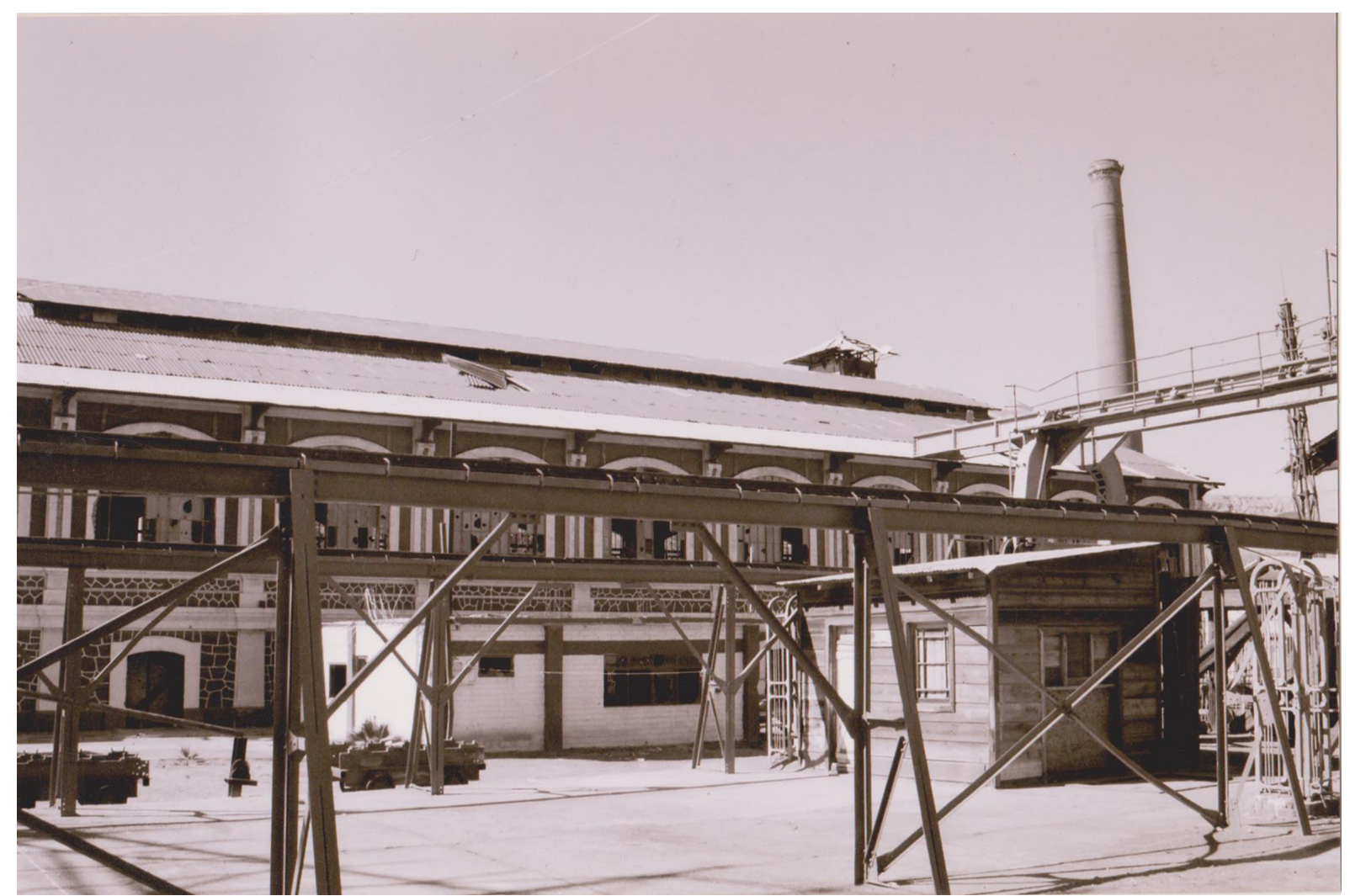

Figura 11. Las primeras 60 mil toneladas de cobre que salieron de Santa Rosalía fueron embarcadas con destino a Swansea en Gales. El primer muelle frente al Golfo de California fue mandado construir por la compañía francesa en 1886; era de madera y tenía una extensión de 250 m de largo. Para 1891 el muelle de madera había sido destruido tres veces a causa de las tempestades. En los próximos años se consolida un dinámico puerto industrial construido de acero y escoria que será capaz de ser en ese tiempo el segundo mayor exportador de cobre del país, hoy estas son sus ruinas. 
nivel. Las vías del tren también determinan el urbanismo que se desarrolló en la zona como parte estructurante de la company town.

La ubicación de los edificios en Santa Rosalía presenta una organización jerárquica ya que se distinguen fácilmente por lo construido en las diferentes áreas. Por ejemplo, las partes altas son siempre las más privilegiadas evitando siempre las inundaciones provocadas por los huracanes.

\section{El periodo de agotamiento del mineral y su nueva oportunidad}

En 1954 la Compagnie Du Boleo cierra operaciones en el poblado al considerar agotados los yacimientos, lo que provoca un éxodo de habitantes. Ante esta situación, el Gral. Agustín Olachea Avilés, Gobernador de Baja California Sur (1946-1956) promovió con el apoyo de la Comisión Nacional de Fomento Minero, la conformación de una empresa de capital mixto, que permitiera la subsistencia de dicho mineral, con lo que se reinicia la explotación minera que concluye en 1972 al haberse agotado los yacimientos de cobre.

Ante esta situación las casas y edificios se abandonan, mientras otras sufren ampliaciones y alteraciones. En 1986 un decreto presidencial declara Santa Rosalía zona de 31 monumentos históricos protegidos por el Instituto Nacional de Antropología e Historia (INAH), y más reciente en 2001 se decide elaborar un estudio con el objetivo principal de conocer los antecedentes históricos, las características arquitectónicas, la traza urbana y los materiales empleados en la construcción de los inmuebles. Si tomamos la definición de que un [...]

[...] paisaje cultural es un ámbito geográfico asociado a un evento, a una actividad o a un personaje histórico, y que contiene, por tanto, valores estéticos y culturales. El proyecto de un parque patrimonial implica garantizar en un determinado paisaje cultural la preservación de sus recursos patrimoniales y al mismo tiempo, ponerlos al servicio de la reactivación económica de la región (Sabate, 2004), [...]

[...] podemos entonces pensar en un posible parque patrimonial para conservar en el territorio los elementos aún de pie de la expresión arquitectónica industrial en esta parte del país. Un claro ejemplo de identidad es la iglesia de Santa Bárbara diseñada presuntamente en 1884 por Gustave Eiffel, la cual se muestra en la Exposición Mundial de París como una manera innovadora de construcción, compuesta de paneles metálicos prefabricados (casas duclos). Los directivos de la compañía la encuentran desarmada en Bélgica en 1895 y es transportada en barco de vela a México, para ser armada en Santa Rosalía en 1897. Se sabe que el origen de la iglesia fue parte de un proyecto de 7 capillas prefabricadas para ser armadas en algún lugar de Africa. En la actualidad se desconoce el paradero de las otras 6.

Hoy en día Santa Rosalía cuenta con la misma cantidad de población que hace cien años, su crecimiento fue determinado por el auge minero que fue la principal actividad económica, en la actualidad hay nuevas compañías mineras extrayendo cobre con nuevas tecnologías y el poblado ha adquirido un nuevo interés a baja escala por la explotación de minerales. Por eso es importante valorizar el urbanismo de sitios como éste, para contribuir al conocimiento del rico patrimonio que queda y que modernizó con su arquitectura la península de California.

\section{Consideraciones finales}

Parece necesario ampliar y difundir las investigaciones sobre la historia reciente del urbanismo en México, ya que se percibe un cierto desconocimiento sobre el surgimiento de este tipo de ciudades, las cuales poseen una identidad incipiente, pero no por ello menos valiosa, en términos de apropiación del espacio, inclusive cuando éstas han sido diseñadas por compañías extranjeras. Es importante nombrar y mostrar los proyectos que hicieron de México un país moderno, ya que muchos de ellos se han convertido en pueblos fantasmas o en ciudades que no reconocen su centro histórico cada vez más despoblado.

El patrimonio industrial es considerado actualmente como un nuevo bien cultural. Como es lógico las regiones y ciudades donde comienza este punto de inflexión son aquellas donde la crisis 
industrial había afectado profundamente y la desesperanza o el fatalismo se había instalado. Se trató en muchos casos de convertir la necesidad en virtud, cobrando interés creciente en políticos locales, movimientos ciudadanos, defensores del patrimonio y la arqueología industrial, con nueva mirada de arquitectos e ingenieros que ponen su objetivo en el territorio urbano (Álvarez, 2011).

Los gobiernos locales y las políticas actuales de preservación deberán encarar el siglo XXI generando nuevos proyectos de regeneración de trazados e instalaciones industriales para poder así garantizar los valores y la identidad urbana, en este caso en particular en la península de Baja California.

Por tanto se requiere crear un proyecto de revitalización de edificios industriales y su puesta en valor para beneficio de toda la comunidad. Desde esta consideración se presentan tres oportunidades para su futuro:

- Mostrar en un eco museo el patrimonio edificado, para resaltar la arquitectura de ese periodo histórico. La recuperación de la identidad del territorio a través de resaltar lo que ya está ahí en ruinas.

- Valorizar la importancia del rico legado de una ciudad para buscar una reactivación económica a través de nuevas y distintas actividades para beneficio de la comunidad, haciendo que se involucren en el mejoramiento paulatino de los espacios.

- Nuevos usos a los terrenos abandonados y restauración de edificios industriales para intentar integrar el espacio deteriorado, mejorándolo tanto en su imagen como en su interacción con la población y el turismo.

\section{Referencias}

Alvarez, M.Á. (2011). "Conservación y restauración del patrimonio industrial en el ámbito internacional". Revista de cultura y ciencias sociales. ABACO 2da época No. 70. Pp 22-39. Gijón [Asturias] España.

Berstein, M. (1964). The Mexican Mining Industry 1890-1950: a Study of Interactions of Politics, Economics and Technology. State University of New York, 1964.

Brown, B. (2009). Introducción e impacto del ferrocarril en el Norte de México. Universidad Autónoma de Ciudad Juárez. pp 231-236. Ciudad Juárez [Chihuahua] México 2009.

Cerutti, M. (2008). "La Enciclopedia de Monterrey". El Norte agringado, pp.125-142. Monterrey [Nuevo León] México: Editorial Grupo Milenio.

Cichowicz, J. (1973). The economic evolution of a mining community: Santa Rosalía, Baja California Sur, Mexico, pp 1-78. The Departament of Geography Oregon State University. Oregon [United States]: Oregon Press.

Garcés, E. (1999). Las ciudades del salitre. Un estudio de las oficinas salitreras en la región de Antofagasta. Universidad del Norte. Santiago [Chile]: Editorial Universitaria.

Garcés, E. (2003). "Las ciudades del cobre". Del campamento de montaña al hotel minero como variaciones de la company town. EURE, 29(88), 131-148. Santiago [Chile].

Jordán, F. (1956). “El otro México". Biografía de Baja California. Instituto de Investigaciones Históricas de Baja California.Tijuana [Baja California] México, 2010.

Korthever, D. (2001). Santa Rosalia and Compagnie du Boleo: The making of a town and company in the porfirian frontier 1885-1900. Irvine [California] United States: University of California Irvine Press.

Padilla, A. (1989). El surgimiento urbano de Santa Rosalía Baja California Sur. pp. 1-16. Tijuana [Baja California] México: Universidad Nacional Autónoma de México, Instituto de Investigaciones Históricas de Baja California. 
Novelo, M.E. (1988). “A history of Santa Rosalía in Baja California”. San Diego [California] United States: San Diego History Center, Institute of History of California.

Quiroz, H. (2008). Ciudades Mexicanas del siglo XX. Ciudad de México : Universidad Nacional Autónoma de México, Facultad de Arquitectura. pp. 23-197.

Romero, M. (1991). "El Boleo, un pueblo que se negó a morir: 1885-1954”. Hermosillo, Editorial Unísono. Centro de estudios mexicanos y centroamericanos. pp.47-110. Ciudad de México 1991.

Sabaté, J. (2004). "Paisajes Culturales. El patrimonio como recurso básico para un nuevo modelo de desarrollo." DU Y OT. Urban 9. Primera época. pp.8-29. Barcelona [Cataluña] España.

Sariego, J.L. (1992). Minería y territorio en México: tres modelos de implantación socio espacial. Fondo de Cultura Económica. ERIA. pp. 197-209. 